\title{
Emerging Technologies in Healthcare: A Tutorial
}

\author{
Matthew N. O. Sadiku, Yogita P. Akhare and Sarhan M. Musa \\ Roy G. Perry College of Engineering \\ Prairie View A\&M University \\ Prairie View, Texas \\ USA
}

\begin{abstract}
Healthcare is a complex system that covers processes of diagnosis, treatment, and prevention of diseases. Technology drives healthcare more than any other force. Technologies in the healthcare change at fast pace from cutting edge to ubiquity. Some of these technologies will change the practice of healthcare and transform our whole approach to illness and health. Although emerging healthcare technologies will not fix all healthcare problems, they can improve the practice, decision making, and management of healthcare. This paper provides a tutorial on emerging technologies in healthcare.
\end{abstract}

Key Words: Emerging technologies in healthcare, Emerging healthcare technologies, Future healthcare technologies.

\section{INTRODUCTION}

We live in the digital age where everything is touched and connected by technology. Our homes, our cars, and our jobs are all connected to technology. Technology is getting better, smaller, and faster. It is becoming more and more in demand in every sector of the economy, particularly in healthcare. The pace of change in healthcare technology is unprecedented, but human nature does not change at these technological timescales. The main stakeholders in healthcare include insurance companies, big pharma, doctors, managers, suppliers, builders, and governments. There have been dramatic technological changes in healthcare. Any sufficiently advanced technology is indistinguishable from magic. Most of these new technologies are modern magic: new pharmaceuticals that change moods, infusion pumps, and robotic keyhole surgery [1].

Health technologies comprise of all the devices, medicines, vaccines, processes, procedures, and systems designed to streamline healthcare operations, lower costs, and enhance quality of care. Technology drives healthcare more than any other force. It is drastically changing and improving healthcare, from anesthetics and antibiotics to MRI scanners and radiotherapy. This technology-driven progress in healthcare is often called Health 2.0. It is well known that hospitals adopt new technologies that enhance their service capabilities and enable them to attract and retain physicians who use the technologies.

\section{CONCEPT OF EMERGING TECHNOLOGY}

Emerging technology (ET) lacks a consensus on what classifies them as "emergent." It is a relative term because one may see a technology as emerging and others may not see it the same way. It is a term that is often used to describe a new technology. A technology is still emerging if it is not yet a "must-have" [2]. An emerging technology is the one that holds the promise of creating a new economic engine and is trans-industrial.

ET is used in different areas such as media, healthcare, business, science, or education. Emerging healthcare technologies cannot be fully exploited without a clinical team to shape the therapeutic response, something hospitals have been able to do over the years with their multidisciplinary clinical workforce. How hospitals and policymakers respond to these emerging technologies will help determine whether hospitals remain at the center of the US healthcare system. Some US hospitals have remarkably responded to these new technologies and adapted their service offerings to incorporate new technologies.

\section{EMERGING HEALTHCARE TECHNOLOGIES}

Emerging technologies in healthcare include information technology, nanotechnology/nanomedicine, biotechnology, cloud computing, Internet of things, augmented/virtual reality, GPS, RFID, voice search, chatbots, social media, blockchain, personalized medicine, biometrics, electronic health records, wearable computing devices, drones, robotics, and artificial intelligence. Of the several emerging technologies, the following examples stand out [3]: 
- Artificial intelligence (AI): This is a field of computer science that is concerned with designing systems to do things that would require intelligence of humans. Today, artificial intelligence is shorthand for any task a machine can perform just as well as, if not better than, humans. AI technologies are now increasingly being been adopted in many areas of the public sector such as education, social interventions, and healthcare. AI in healthcare refers to the application of AI technology in the diagnosis and treatment of patients. AI is being applied in healthcare to review mammograms, monitor early stage heart disease, and enable accurate decision-making among medical practitioners. Today, AI is already being used in medicine in several other areas such as decision support systems, laboratory information systems, robotic surgical systems, therapy, and reducing human error [4].

- 3D Printing: 3D printing (also known as additive manufacturing (AM) or rapid prototyping (RP) was invented by Charles Hull in the early 1980s. Since then it has been used in manufacturing, automotive, electronics, aviation, aerospace, consumer products, education, entertainment, medicine, space missions, the military, and chemical and jewelry industries. It is a technology perfectly tailored for the healthcare industry. It offers a range of precision healthcare solutions, including tissue and organ fabrication, creation of customized prosthetics, implants, and anatomical models, drug delivery, and testing, as well as in clinical practice. Benefits of 3DP in healthcare include the customization and personalization of medical products, drugs, and equipment; cost-effectiveness; increased productivity; the democratization of design and manufacturing; and enhanced collaboration. Hospitals could potentially create items on demand, and this would significantly alter the healthcare supply chain [5].

- Augmented/Virtual Reality: Virtual reality (VR) is a highly interactive, computer-based multimedia environment in which the user becomes the participant in a computer-generated world. For example, surgical students can use virtual overlays of the circulatory system to help direct them during procedures. Billing agents can use "smart glasses" to see patient insurance and billing information when they are away from their computers. It can help reduce the amount of anxiety a patient is feeling before and after surgery. It can be used to train surgeons in a realistic and low risk simulated environment. It offers therapeutic potential and rehabilitation for acute pain and anxiety disorders [6].

- Nanomedicine: Nanomedicine, a marriage of nanotechnology and medicine, is taking the place of nanotechnology in the fight against unmet diseases. Nanotechnology is the science of small things or the manipulation of matter on an atomic or molecular scale. Nanomedicine is essentially the medical application of nanotechnology to the diagnosis, management, and treatment of disease. It is regarded as one of the most promising technologies of the 21 st century. It seeks to manufacture drugs and other products that are packaged into nanoscale systems for improved delivery. The most prominent area of nanomedical research and drug approvals is cancer treatment. The application of nanomedicine, particularly in cancer treatment, promises to have a profound impact on health care. Medications can be more efficiently delivered to the site of action using nanotechnology [7].

- Robotics: This deals with the design, construction, operation, and application of robots. Robots are becoming an integral part of the healthcare toolkit. For example, imagine a robot that can draw a patient's blood with no mistakes and no pain. Robots play an important role in healthcare as they can improve diagnosis, lower the number of medical errors, and improve the overall quality and effectiveness of healthcare delivery. They hold the promise of addressing major healthcare issues in surgery, diagnostics, prosthetics, physical and mental therapy, monitoring, and support. Robots have the potential to provide assistance to healthcare providers in daily caregiving tasks. Transport, telemedicine, and service robots in healthcare promise to create a new level of quality healthcare by providing experts to patient. A wide range of robots is developed to serve different purposes within the healthcare environment. This results in various kinds of healthcare robots such as surgical robots, logistics robots, disinfectant robots, cleaning robots, pill robots, laboratory robots, rehabilitation robots, nursing robots, telepresence robots, therapy robots, assistive robots, robotic prosthetic limbs, diagnostics robots, and many other types $[8]$.

- Cloud Computing: This is an on-demand and self-service Internet infrastructure that offers large scalable computing and storage, data sharing, on-demand anytime and anywhere access to resources. The healthcare industry has been hesitant in embracing the cloud computing because of the concern of data privacy and integrity. Cloud computing is changing the way healthcare providers (doctors, clinics, and hospitals) deliver services to their patients. Healthcare providers are increasingly facing keen competition and are compelled do more for less. They are rapidly turning to the cloud to address the business and patient needs. On the patient side, people are accustomed with managing their own healthcare needs. Application areas include emergency healthcare, home healthcare, assistive healthcare, telemedicine, storage, sharing and processing of large medical resources [9].

- Internet of Things (IoT): This allows all entities to be connected to each other through wired or wireless communication means. IoT has been gaining popularity rapidly since its inception into the IT world and is being used in healthcare, education, gaming, finance, transportation, and several more. The healthcare industry is among the fastest to adopt the Internet of things. Applications of IoT in healthcare are numerous, ranging from remote monitoring to smart sensors and medical device integration. The applications benefit patients, families, nurses, and physicians. IoT healthcare is applicable in many medical instruments such as ECG monitors, glucose level sensing, and oxygen concentration detection. It has been long predicted that IoT healthcare will revolutionize the healthcare sector in terms of social benefits, penetration, accessible care, and cost-efficiency [10]. A typical IoT-based healthcare system is shown in Figure 1 [11]. 
- Blockchain: This technology consists of a shared or distributed database used to maintain a growing list of transactions, called blocks. With blockchain (BC), transaction records are stored and distributed across all network participants rather than at a central location. Blockchain in health care will be in clinical trial records, regulatory compliance, and medical records. The technology can help medical practitioners make better and more accurate diagnoses and prescribe more effective treatments. The goal of $\mathrm{BC}$ is to give patients and their providers one-stop access to their entire medical history across all providers. Blockchain is able to securely, privately and comprehensively track patient health records. It makes electronic medical records more efficient, disintermediated, and secure. It also makes health information exchanges (HIE) more secure, efficient, and interoperable [12].

- Social media: Social media refers to Internet-based and mobile-based tools that allow individuals to communicate with things. This is king in healthcare marketing. The Internet has empowered individuals to share health information and interact using social media. Social media are basically web-based tools used for computer-mediated communication. It is a powerful tool that healthcare professionals can use to communicate and interact with patients. It has become an undeniable force that healthcare industry must reckon with. Although social media is still evolving, it has made a profound impact on the healthcare industry [13].

Some of these technologies are illustrated in Figure 2 [14]. Besides these, there are also many other different technological inventions being made to help in difficult situations.

\section{APPLICATIONS OF EMERGING HEALTHCARE TECHNOLOGIES}

Emerging technologies are usually more accessible, less expensive, and easier to learn than their predecessors. The technologies can be used for prevention of diseases, diagnostics, effective healthcare delivery, rehabilitation, improving quality of life for patients, reducing morbidity and fatality rates, and enhancing healthcare access [15]. Emerging technologies have been noted as potential mechanisms for reducing medication errors. An amalgamation of these technologies will be needed to have an end-toend solution for emerging healthcare applications.

The emerging technologies presented above can be applied to every aspect of healthcare. Some popular areas include the following [16]:

- Regenerative Medicine: This basically involves culturing and grafting human tissues, both artificial and cultured. Remarkable advances have already been made in culturing and grafting human cells to repair burn damage. Regenerative medicine has the potential to heal or replace tissues and organs due to age, disease, damage, trauma, or congenital defects. This field tends to regenerate damaged tissues and organs in the body by stimulating previously irreparable organs to heal themselves [17].

- Remote patient Monitoring: A rapidly emerging area of service in healthcare is in remote monitoring of patients with some degree of unstable clinical risk. It involves having small sensors placed on body of a patient to monitor various health parameters like blood pressure, heartbeat, temperature and prolonged electrocardiogram. The potential advantages of remote monitoring of implantable devices could include timely monitoring of clinical events and symptoms. The time spent by medical staff be saved [18].

- Personalized Healthcare: Personalized healthcare is healthcare tailored to suit individual patients. It is one of the areas that benefit from IoT. Emerging technologies that provide personalized healthcare services to patients include [19]: (1) Pattern recognition methods for prediction and diagnosis of diseases, (2) Body sensor networks. (3) Algorithms for the analysis of patient-specific physiological signals, (4) Ontologies and context-based electronic health records (EHRs), (5) Modelling of physiological systems, (6) Monitoring and treatment support tools for chronic diseases, (7) Patient-specific multiscale modelling, (8) Integrated e-health solutions. Personalized healthcare offers numerous benefits to medical professionals and patients especially elderly people. It makes it possible for doctors and medical staff to monitor patients remotely and for patients to receive instant treatment [20]. They can obtain patient's body parameters remotely and at regular interval. The IoT devices gather information from patients and share the information with medical professionals. Also, 3D printed pharmaceuticals could accelerate mass customization of medicine and innovation.

- Electronic Health Records (HER): The electronic health record (EHR) is a digital record of a patient's health history. EHR may be made up of records from many sources such as hospitals, providers, clinics, and public health agencies. Health records continue to evolve as a result of technology. Any changes in documentation of care have a significant impact on nursing practice. EHR is available $24 / 7$ and has built-in safeguards to assure patient health information confidentiality and security [21]. Emerging technologies have been recommended as potential mechanisms for reducing medication errors.

Other applications include surgery, cancer treatment, healthcare education, and wearable devices. 
International Journal of Advances in Scientific Research and Engineering (ijasre), Vol 5 (7), July-2019

\section{BENEFITS AND CHALLENGES}

The benefits that emerging technologies have brought to healthcare include [22]:

- Reducing healthcare costs

- Greater patient care

- Avoiding preventable deaths

- Improving quality of life

- Improving public health

- Reducing healthcare waste

- Improving efficiency and quality of care

- Developing new drugs and treatments

A growing number of healthcare organizations are embracing new technologies to increase their efficiency and effectiveness. Adopting these technologies will help healthcare facilities provide higher quality patient care at a lower cost.

While these technologies offer countless benefits, there are some major concerns and drawbacks which include:

- Some patients may not be savvy with technology.

- Implementing a new technology may be a prohibitive and uphill task.

- When technology fails to work, it can be frustrating.

- A single mistake in using technology can cause disastrous consequences.

- New technology can be complex or expensive, causing a slow rate of adoption.

- Security and privacy risks are of paramount importance.

- Medical information is very sensitive; its misuse could have serious consequences.

- Shrinking budgets inhibits innovations.

- Healthcare technology providers are mostly interested in their bottom line.

In the future there will remain an enduring distinction between safety and security. The future trends should include tightening the culture of technology development. Despite their advantages and disadvantages, emerging technologies really make our lives easier.

\section{CONCLUSION}

Frankly speaking, healthcare has no end of problems: we all want and expect better care, costs are rising, and performance is declining, we live living longer with chronic illness, etc. If we want healthcare to improve in the future, we must continuously plan for it.

Future technological innovations (new drugs, new treatments, new devices, etc.) will keep transforming healthcare. Since technology drives healthcare, the fundamental problems of wellbeing, health and happiness, will remain. We need to be aware of the drivers, align with them, and work with them to ensure the best outcomes for society.

The emerging healthcare technologies discussed in this paper have several things in common: large, fixed-cost expenditures to acquire the technologies and a need for highly trained clinical operators [15]. More information on emerging health technologies can be found in the books in [23-25]. 


\section{REFERENCES}

[1] H. Thimbleby, "Technology and the future of healthcare," Journal of Public Health Research, vol. 2, no. 3, December 2013.

[2] M. Halaweh, "Emerging technology: What is it?” Journal of Technology Management \& Innovation, vol. 8, no. 3, 2013, pp. 108-115.

[3] “5 Technology trends impacting healthcare,”March 2018, https://cbcommunity.comcast.com/browse-all/details/5-technology-trends-impacting-health-care.

[4] M. N. O. Sadiku, T. J. Ashaolu, and S. M. Musa," Artificial intelligence in medicine: A primer," International Journal of Trend in Research and Development, vol. 6, no. 1, Jan.-Feb. 2019, pp. 270-272.

[5] M. N. O. Sadiku, J. Foreman, S. M. Musa, “3D Printing in healthcare,” International Journal of Scientific Engineering and Technology, vol. 7, no. 7, July 2018, pp. 65-67.

[6] M. O. Onyesolu1 and F. U. Eze, "Understanding virtual reality technology: Advances and applications," https://www.semanticscholar.org/paper/Understanding-Virtual-Reality-Technology\%3A-Advances-OnyesoluEze/12c4294ce620ec062e8dad1acb738b7cc9d211ba

[7] M. N. O. Sadiku, T. J. Ashaolu, and S. M. Musa," Nanomedicine: A primer," International Journal of Trend in Research and Development, vol. 6, no. 1, Jan.-Feb. 2019, pp. 267-269.

[8] M. N. O. Sadiku, Y. Wang, S. Cui, and S.M. Musa,” Healthcare robotics: A primer,” International Journal of Advanced Research in Computer Science and Software Engineering, vol. 8, no. 2, Feb. 2018, pp. 26-29.

[9] M. N. O. Sadiku, S. R. Nelatury, and S.M. Musa, "Cloud computing in healthcare," Journal of Scientific and Engineering Research, vol. 5, no. 9, 2018, pp. 202-205.

[10] M. N. O. Sadiku, S. Alam, and S.M. Musa, "IoT for healthcare," International Journal of Electronics and Communication Engineering, vol. 5. no. 11, November 2018, pp. 5-7.

[11] M. M. Dhanvijay and S. C. Patil, "Internet of things: A survey of enabling technologies in healthcare and its applications," Computer Networks, vol. 153, 2019, pp.113-131.

[12] M. N. O. Sadiku, K. G. Eze, and S.M. Musa, "Block chain technology in healthcare," International Journal of Advances in Scientific Research and Engineering, vol. 4, no. 5, 2018, pp. 154-159.

[13] M. N. O. Sadiku, N. K. Ampah, S. M. Musa, "Social media in healthcare," International Journal of Trend in Scientific Research and Development, vol. 2, no. 5, June/July 2018, pp. 665-668.

[14] A. Datta,’Top eight disruptive technologies and how they are relevant to geospatial," 2018, https://www.geospatialworld.net/blogs/top-disruptive-technologies-relevant-geospatial/

[15] M. Ilyas, "Emerging technologies for healthcare," Proceedings of International Symposium on High Capacity Optical Networks and Enabling Technologies, 2008, pp. 15-18..

[16] J. Goldsmith, "Technology and the boundaries of the hospital: Three emerging technologies," Health Affairs, vol. 23, no. 6, November/December 2004, pp. 149-156.

[17] A. S. Maoa and D. J. Mooney, "Regenerative medicine: Current therapies and future directions," PNAS, vol. 112, no. 4, November 2015, pp. 14452-14459.

[18] D. Panescu, "Wireless communication systems for implantable medical devices," IEEE Engineering in Medicine and Biology Magazine, March/April 2008, pp. 96-101.

[19] M. Akay et al., "Emerging technologies for patient-specific healthcare," IEEE Transactions on Information Technology In Biomedicine, vol. 16, no. 2, March 2012, pp. 185-189.

[20] M. M. Alam et al., "Survey on the roles of communication technologies in IoT-based personalized healthcare applications," IEEE Access, vol. 6, 2018, pp. 36611-36631.

[21] C. Huston, "The impact of emerging technology on nursing care: Warp speed ahead," The Online Journal of Issues in Nursing, vol. 18, no. 2, May 2013.

[22] B. Banova, "The impact of technology in healthcare," June 2019, https://www.aimseducation.edu/blog/the-impact-of-technology-on-healthcare/

[23] S. Jones and F. M. Groom (eds.), Information and communication Technologies in Healthcare. Boca Raton, FL: CRC Press, 2012.

[24] J. M. Boyce, Measuring Healthcare Worker Hand Hygiene Activity: Current Practices and Emerging Technologies. Cambridge University Press, 2015.

[25] J. A. Jacko (ed.), The Human-Computer Interaction Handbook: Fundamentals, Evolving Technologies, and Emerging Applications. Boca Raton, FL: CRC Press, $3^{\text {rd }}$ edition, 2012. 


\section{AUTHORS}

Matthew N.O. Sadiku is a professor in the Department of Electrical and Computer Engineering at Prairie View A\&M University, Prairie View, Texas. He is the author of several books and papers. His areas of research interests include computational electromagnetics and computer networks. He is a fellow of IEEE.

Yogita P. Akhare is a doctoral student at Prairie View A\&M University, Prairie View, Texas. Her research interests include machine drives and nanotechnology.

Sarhan M. Musa is a professor in the Department of Engineering Technology at Prairie View A\&M University, Texas. He has been the director of Prairie View Networking Academy, Texas, since 2004. He is an LTD Sprint and Boeing Welliver Fellow.

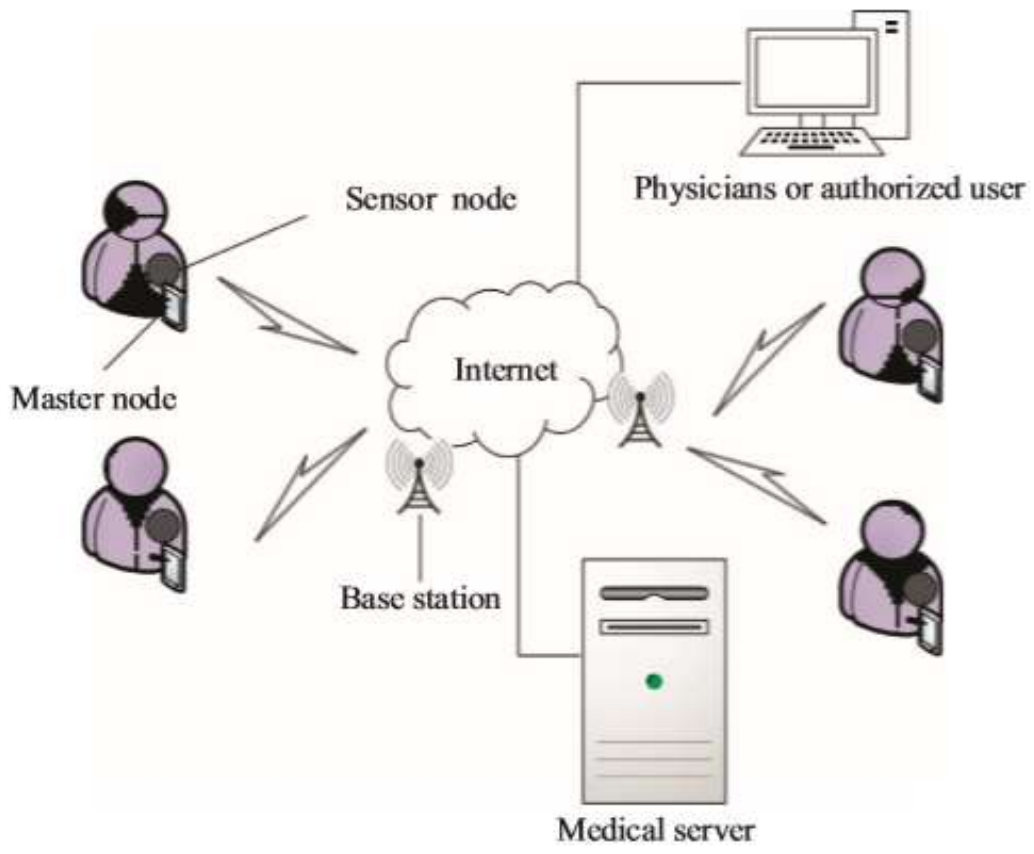

Figure 1: A typical IoT-based healthcare system [11].

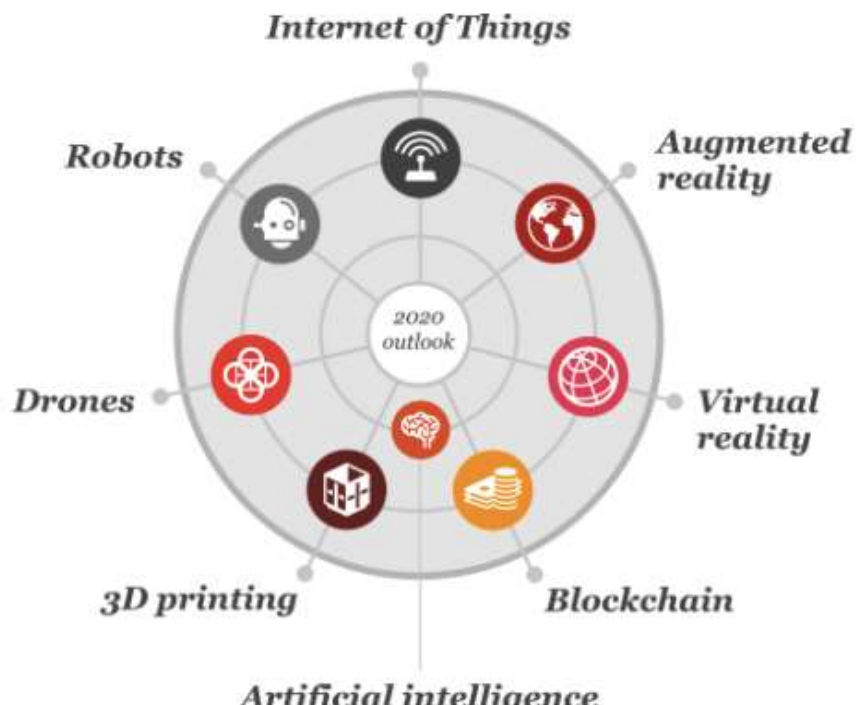

Figure 2: Some emerging healthcare technologies [14]. 